\title{
Modifikation der Disc-Elektrophorese zur direkten Gewebselektrophorese von Kryostatschnitten bioptisch gewonnener Gewebeproben ${ }^{1}$ )
}

\author{
Von F. Mittelbach, W. Anti, und K. Hamburger ${ }^{2}$ ) \\ Aus der II. Medizinischen Klinik der Universität München (Direktor Prof. Dr. Dr. G. Bodechtel)
}

(Eingegangen am 26. November 1968)

\author{
Herrn Prof. Dr. Dr. G. Bodechtel in dankbarer Verebrung zum 70. Geburtstag
}

Es wird eine Modifikation der Disc-Elektrophorese beschrieben, wobei Gewebeproben bioptischen Materials (Muskel) in Form von Kryostatschnitten direkt in das System eingebracht werden. Durch das diskontinuierliche Puffersystem sowie die Verschiedenheit von Sammelgel einerseits und Trenngel andererseits wird gewissermaßen Extraktion und elektrophoretische Trennung in einem Arbeitsgang durchgeführt. Die Versuchsanordnung ist einfach und gestattet zuverlässige und scharfe Trennungen der löslichen Proteinfraktionen. Die Reproduzierbarkẹt ist gut.

\section{A modified disc electrophoresis method for the direct tissue electrophoresis of cryostate biopsy samples}

A distinct separation of tissue proteins is achieved by modified disc electrophoresis technique. Frozen tissue sections are applied directly to the gel. The separation is quick and convenient, and there is high reproducibility.

Die elektrophoretische Auftrennung von Gewebsproben hat zunehmend an Bedeutung und Interesse gewonnen. Die hierzu notwendigen Gewebsextrakte erfordern zu ihrer Gewinnung größere Gewebsmengen, so $\mathrm{da} ß$ bei bioptisch gewonnenem Material diese Untersuchungsmethoden nur begrenzt anwendbar sind. In besonderem Maße gilt dies für die Skeletmuskulatur, da die bei der Biopsie gewonnenen Gewebsproben auch für gleichzeitige histologische und histochemische Untersuchungen verwendet werden, und somit sparsamste Verwendung angezeigt ist. Zudem findet man eine große Reihe von Beeinflussungen bei Extraktelektrophoresen, wie z. B. unterschiedliche Auftrennung bei Anwendung verschiedener Elektrodenpuffer (1) oder unterschiedliche Löslichkeit der Proteine je nạch Zusammensetzung und Ionenstärke des bei der Extraktion verwendeten Puffers. Eine Möglichkeit, die Proteintrennung mit den geringen vorhanderien Substanzmengen durchzuführen, bietet die unmittelbare Einbringung von Gewebsschnitten in eị geeignetes Elektrophoresesystem.

Einen Forstschritt brachte hier die von RAYMOND und Weintraub (2), sowie Davis (3) und Ornstein (4) entwickelte Elektrophorese in Polyacrylamidgel.

Die Vorzüge gegenüber anderen Gelen bestehen einmal in der beliebig $z u$ variierenden Porengröße und in seiner Transparenz, zum anderen in der mechanischen und chemischen Stabilität über weite pH- und Temperaturbereiche und schließlich in dem in Folge des elektroneutralen Gelcharakters fast völligen Fehlen einer Elektroosmose. Die in diesem Trägermedium erhaltenen

\footnotetext{
1) Mit Unterstützung der Friedrich-Baur-Stiftung, München und der Deutschen Forschungsgemeinschaft.

2) Diese Arbeit enthält wesentliche Teile der Inaugural-Disserta-
} tion von $\mathrm{K}$. Hamburger.
Resultate zeigten neben einer überraschenden Vielfalt des Proteinmusters eine ausgezeichnete Trennschärfe der einzelnen Proteinbanden, sowie eine gute Reproduzierbarkeit.

Die von Davis und ORNSTEIN entwickelte DiscMethode, welche den schon 1957 von Poulik (5) entdeckten elektrophoretischen Effekt eines diskontinuierlichen Puffersystems ausnützt, bringt zudem noch den Vorteil der einfachen und schnellen Ausführung einer Polyacrylamidgel-Elektrophorese mit geringstem Materialaufwand. Diese Eigenschaften, sowie die für unsere $Z$ wecke sehr dienliche apparative Anordnung veranlaßten uns, die Disc-Elektrophorese für unsere Untersuchungen $\mathrm{zu}$ übernehmen und unseren Bedürfnissen entsprechend zu modifizieren.

\section{Methodik}

\section{Prinzip der Disc-Elektrophorese}

Die Trennkapazität der von Davis und ORNSTEIN entwickclten Disc-Elektrophorese wird nach der Theorie von ORNSTEIN durch die verschiedenen Diskontinuitäten $(\mathrm{pH}$ und Ionenstärke der verwendeten Puffer, Porenweite der Gele) des Elektrophoresemilieus erreicht. Die elektrophoretische Trennung findet in Glasröhrchen statt, in denen zwei verschiedene Polyacrylamidgelschichten zur Polymerisation gebracht wurden.

Für die großporigen Gelabschnitte (Sammelgel, 2,5\%) wird ein $0,5 \mathrm{M}$ Tris-Phosphat-Puffergemisch, $\mathrm{pH} 6,9$, und für das kleinporige 7 proz. Trenngel ein 3,0 M Tris-HCl-Puffer, $\mathrm{pH} \mathrm{8,9,} \mathrm{vcr-}$ wendet. Der Elektrodenpuffer besteht aus $0,05 \mathrm{M}$ Tris- $0,385 \mathrm{M}$ Glycin $(\mu=0,11), \mathrm{pH} 8,3$. Die Puffersysteme sind so gewählt, $\mathrm{da} \beta$ beim Anlegen der Spannung eine Ionenfront, bestehend aus schnell wandernden Chloridionen und langsamer wandernden Glycinionen, das großporige Gel durchläuft und sich dic dort befindlichen Proteine des Untersuchungsmaterials in der Schicht zwischen diesen beiden Ionenarten ansammeln und mitwandern. Die Proteine creichen das kleinporige Trenngel maximal konzentriert in einer extrem dünnen Startzonc. Auf Grund des pliWechsels erreicht die Chlorid-/Glycin-Ionenschicht hier eine größere Beschleunigung als sämtliche Proteine und eilt nun vor- 
aus. Sie hinterläßt im Trenngel einen Bcreich mit relativ konstantem pH-Wcrt und Spannungsgradienten, in dem die Proteinc ciner norınalen Trägerelektrophorese nach ihrer clektrophoretischen Beweglichkeit, kombinicrt mit eincr Gelchromatographie nach Molekülgestalt und -größe untcrworfen werden.

\section{Elektroplsoreseapparal}

Dic Geräte, die für die Disc-Elektrophorese gebraucht werden, sind verhältnismäßig einfach und lassen sich leicht ohne größere Kosten selbst herstellen.

$\Lambda$ nfangs verwendeten wir das bei (6) beschricbene Gerät. Bei den ersten Auftrennungen beobachteten wir eine relativ starke Wärmeentwicklung in den Röhrchen mit teilweiser Verflüssigung des Sammelgels. $\mathrm{Da}$ aber eine Kühlung in dieser Apparatur auf gewisse technische Schwierigkeiten stieß, fertigten wir ein zylindrisches Disc-Elcktrophorese-Gerät an, welches unseren Forderungen nach einer Kühlung der Gelröhrchen entsprach. Dies erreichten wir dadurch, daß wir den Abstand zwischen Kathodenpuffergefäß, Anodenträger (der gleichzeitig zur senkrechten Führung der Röhrchen dient) und Oberrand des Anodenpuffergefäßes so gering wie möglich hielten, so daß die Röhrchen zum größten Teil in das Anodenpuffergefäß hineinragten.

Füllt man nun das untere Puffergefäß mit Elektrodenpuffer von $0-5^{\circ}$ (Kühlschranktemperatur) bis $0,5 \mathrm{~cm}$ unter den Rand, so tauchen die Gelröhrchen einschließlich der Probenschicht völlig in dic kaltc Pufferlösung ein. Sollte eine stärkere Kühlung notwendig sein, so kann man dies leicht durch Einbau einer Kühlschlangc aus Glas in das untere Puffergefäß oder dadurch, daß man das untere Puffergefäß in eine Kältemischung aus Eis und Wasser stellt, erreichen.

Folgende Lösungen kamen entsprechend den Angaben von Davis und OrNstein zur Verwendung. Lediglich für das großporige Gel wurde davon abweichend statt- Tris-HCl-Puffer' ein Tris-Phosphat-Puffer, $\mathrm{pH} 6,9$ benützt (7).

Lösungen

A 3,0 M Tris-HCl-Puffer, $\mathrm{pH} 8,9$

$\begin{array}{ll}\text { 1N } \mathrm{HCl} & 48,0 \mathrm{ml} \\ \text { Tris } & 36,6 \mathrm{~g} \\ \left.\text { TEM(ED }{ }^{3}\right) & 0,23 \mathrm{ml}\end{array}$

dest. Wasser auf $100 \mathrm{~m} l$

B 0,5 M Tris-Phosphat-Puffer, $\mathrm{pH} \mathrm{6,9}$

$\begin{array}{ll}1_{\mathrm{M}} \mathrm{H}_{3} \mathrm{PO}_{\downarrow} & 25,6 \mathrm{~m} / \\ \text { Tris } & 5,7 \mathrm{~g} \\ \text { TEMIED } & 0,46 \mathrm{~m} l\end{array}$

dest. Wasser auf $100 \mathrm{~m} /$

C Acrylamid $28,0 \mathrm{~g}$

BIS $\quad 0,735 \mathrm{~g}$

dest. Wasser auf $100 \mathrm{~m} /$

D Acrylamid $10,0 \mathrm{~g}$

BIS 2,5 $\mathrm{g}$

dest. Wasser auf $100 \mathrm{ml}$

E Riboflavin $4,0 \mathrm{mg} / 100 \mathrm{ml}$

F Saccharose $40,0 \mathrm{~g} / 100 \mathrm{ml}$

G Ammoniumperoxydisulfat $140 \mathrm{mg} / 100 \mathrm{~m} /$

Elektrodenpuffer (Tris-Glycin, $\mathrm{pH} 8,3$ ):

Glycin $\quad 28,8 \mathrm{~g}$
Tris $\quad 6,0 \mathrm{~g}$
dest. Wasser auf 21

Für die Elektrophoresc rerden die gereinigten Glasröhrchen (Länge $10 \mathrm{~cm}$, innerer $\varnothing 5 \mathrm{~mm}$, bei 2,0 und $3,5 \mathrm{~cm}$ markiert) mit dem markierten Ende nach unten in zur Hälfte durchbohrte Gummistopfen gesteckt und mit einer langen Hohlnadel nochmals mit dem Gclpuffer B ausgespült. Die erforderlichen Polymerisationsmischungen werden kurz vor Gebrauch aus den Grundlösungen A-G hergestellt.

3) TEMED $=\mathrm{N}, \mathrm{N}, \mathrm{N}^{\prime}, \mathrm{N}^{\prime}$-Tetramethyläthylendiamin; BIS = $N, N^{\prime}$-Methylenbisacrylamid.
Für das großporige Sammelgel:

1 Teil B, 1 Teil E, 2 Teile D, 4 Teile F ( $v / v)$.

Für das kleinporige Trenngel:

1 Tcil A, 1 Teil Wasser, 2 Teile C, 4 Teile G (v/v).

Bis zur Marke 2,0 cm werden die Röhrchen nur mit großporigem Probengel aufgefüllt und nach Uberschichtung mit einem Tropfen Wasser etwa $20 \mathrm{Min}$. lang zur Polymerisation zwischen zwei parallel verlaufende Leuchtstoffröhren gestellt (in der Originalmethode ist UV-Licht empfohlen, unserer Erfahrung nach erfolgte die Polymerisation mit Neonleuchten sogat besser). Diese unterste Gelschicht dient der Abdeckung der Gewebeprobe gegen den Elektrodenpuffer. Nun trägt man $0,02 \mathrm{ml}$ einer Lösung aus Gelpuffer B und Saccharoselösung $(1: 4, v / v)$ auf das Gel auf.

Das Gewebe, das untersucht wurde, stammte von vier verschiedenen Patienten, welche keinerlei Anzeichen einer pathologischen Muskelveränderung besaßen, was durch die vorgenommenen histologischen und histochemischen Untersuchungen bestätigt wurde. In allen vier Fällen handelte es sich um bioptisch erhaltene Muskelstücke aus dem M. tibialis anterior, sofort in flüssigem Stickstoff eingefroren und in der Kühltruhe bei $-60^{\circ}$ aufbewahrt. Man schneidet im Kryostaten von dem Muskelstück eine Anzahl $40 \mu \mathrm{m}$ dicker Schnitte. Bei einer Anschnittfläche von ungefähr $25 \mathrm{~mm}^{2}$ sind etwa 2 Schnitte für ein Röhrchen ausreichend (8).

Mit einem kleinen, $4 \mathrm{~mm}$ breiten Spatel werden die benötigten Schnitte aufgenommen und so zusammengefaltet, da $B$ sie, ohne an der Wand des Röhrchens anzustreifen, in die $0,02 \mathrm{ml}$ PufferSaccharose-Schicht eingebaut werden können. Nach dem Abstreifen erfolgt durch Hin- und Herbewegen des Spatels eine grobe Zerkleinerung des Muskelgewebes, damit es sich möglichst homogen und eben auf die Geloberfläche auflegt. Noch schwebende größere Teilchen können mit der Spatelspitze durch leichtes Andrücken an den Gelboden fixiert werden. Sind alle acht Röhrchen auf diese Weise gefüllt worden, schichtet man Sammelgel bis zur Marke $3,5 \mathrm{~cm}$ darüber und läßt 20 Min. polymerisieren. Als letztes erfolgt die Beschickung mit dem kleinporigen Trenngel, dessen Polymerisation nach etwa 30 Min. beendet ist.

Die so vorbereiteten Röhrchen werden in den ElektrophoreseApparat eingeführt. Es wird eine Stromstärke von $2,5 \mathrm{~mA} / \mathrm{Gel}$ eingestellt. Ein Zusatz von $0,5 \mathrm{ml}$ einer 0,001 proz. wäßr. Bromphenolblaulösung pro $100 \mathrm{ml}$ Pufferlösung (3) zum Kathodenpuffer dient der Sichtbarmachung der wandernden Pufferfront. Nach etwa 110 Min. ist die Auftrennung beendet. Die Puffergrenze durchlief in allen unseren Versuchen die Gelsäulen in den einzelnen Röhrchen gleichmäßig schnell, so daß eine gute Vergleichsmöglichkeit der Ergebnisse gewährleistet ist. Nach Beendigung der Auftrennung werden die Gelsäulen durch Umfahren mit einer Hohlnadel bei gleichzeitigem Einspritzen von.Glycerin aus den Glastöhrchen entfernt und in einer Lösung von $1 \%$ Amidoschwarz in 7proz. Essigsäure 15-60 Min. gefärbt.

Die Entfärbung exfolgte elektrophoretisch in 7proz. Essigsäure anfangs mit unserem Disc-Elektrophorese-Gerät in Auftrennrichtung. $\mathrm{Da}_{\mathrm{a}}$ es jedoch nicht möglich war, auf diese Weise die relativ starke Hintergrundfärbung der Gele selbst bei Anwendung sehr langer Entfärbezeiten 2,5-5,0 mA/Gel bis zu $24 \mathrm{Stdn}$.) zu verringern, und Versuche mit anderen Proteinfarbstoffen wie Coomassie Brilliant Blau R-250 (9, 10, 11), Procion Brilliant Blau RS (12,7), Bromphenolblau, Lichtgrün und Azokarmin B keinerlei Verbesserung brachten, entschlossen wir uns zu einer elektrophoretischen Entf̈ärbung der Gele senkrecht zur Auftrennungsrichtung (13-16).

Hierzu fertigten wir aus Plexiglas eine kühlbare Apparatur zur Untergrund-Entfärbung von Gelen in horizontaler Lage (Abb. 1). Die Entfärbekammer ist von einem Kühlmantel umgeben, in dem man je nach Erwärmung Kühlwasser zirkulieren lassen kann. Eine Trennwand teilt die Entfärbekammer in zwei Kompartimente.

Diese Trennwand besteht aus 3 Teilen:

1. Der U-förmige Rahmen von 5-6 mm Dicke nimmt die Gelsäulen auf.

2. Das vor ihm befestigte Gitter verhindert ein Herausfallen der Gelsäulen. 
3. Die an der Hinterfront des U-Rahmens befestigte Glasfritte $\mathrm{P}_{4}$ (Firma Schott, Mainz) erfüllt die Aufgabe einer Diffusionsmembran.

Die parallel zueinander stehenden Kobleelektroden sind am Deckel befestigt und tauchen beiderseits der Trennwand parallel $z u$ ihr in die beiden Kompartimente der Entfärbekammer ein.

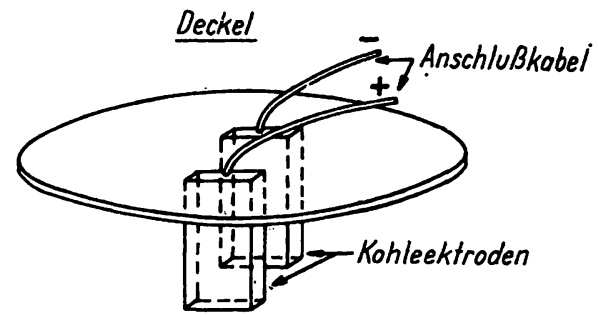

\section{Trennwand}

Gele
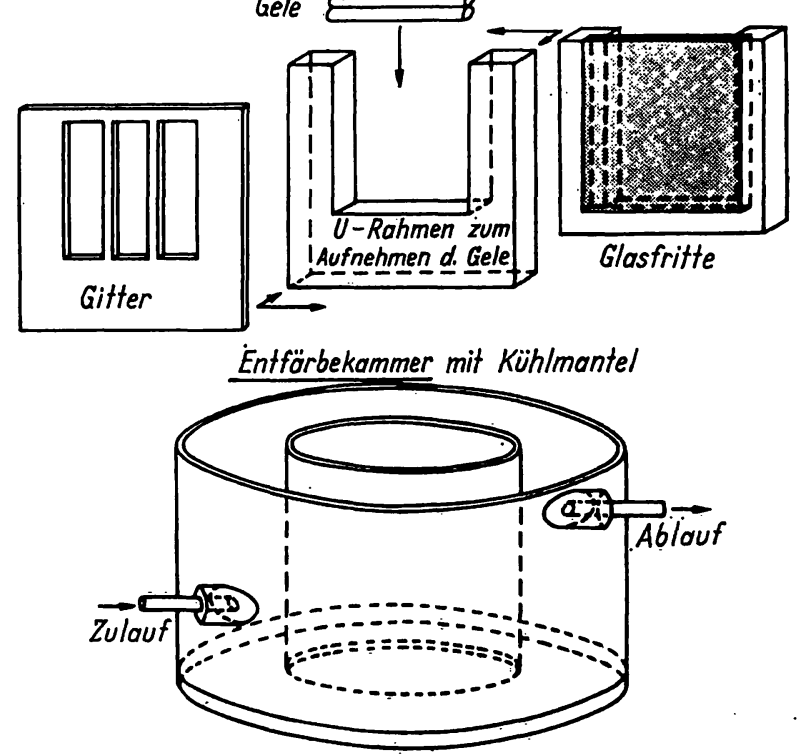

Abb. 1

Gerät zur Untergrund-Entfärbung von Disc-Elektrophorese-Säulen in. horizontaler Lage

Eine 3stdg. elektrophoretische Entfärbung der Gelsäulen bei $20-25 \mathrm{~mA} / \mathrm{Gel}$ (14) brachte eine wesentliche Verringerung der Hintergrundfärbung. Völlig ließ sich diè Untergrundfärbung jedoch auch mit dieser orthogonalen Entfärbemethode nicht beseitigen. Die Ursachen hierfür sind uns nicht bekannt.

\section{Ergebnisse}

Die Auftrennung der Muskelproteine des Menschen gab ein in den Grundzügen sich regelmäßig wiederholendes Bild, was die einwandfreie Reproduzierbarkeit unserer Methode unterstreicht (Abb. 2). Das zum Teil bis zu 4 Monate in der Kühltruhe aufbewahrte Muskelgewebe zeigte keine Änderung des Proteinmusters bei der elektrophoretischen Trennung gegenüber frischen Geweben. Dies entspricht den Ergebnissen von DiETz und Lubrano (17), die zeigen konnten, daß selbst nach mehrmonatiger Lagerung von Seren bei $-65^{\circ}$ der Verlust von Enzymaktivität nach disc-elektrophoretischer Auftrennung nur etwa 10\%-betrug und das Proteinmuster unverändert blieb. Während der Elektrophorese läßt sich mit bloßem Auge eine bräunliche Eiweißbande erkennen, die nach dem Abschalten des Stromes ungefähr bis zur Mitte des Trenngels ge-

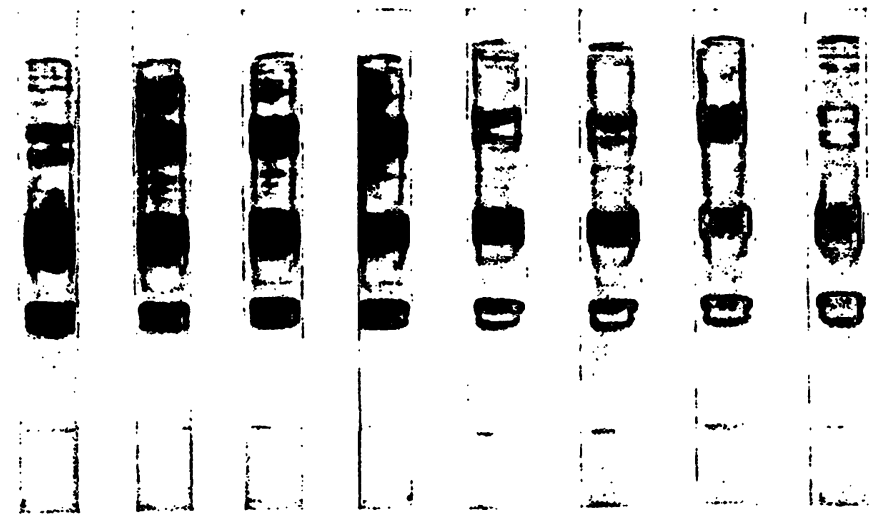

Abb. 2

Versuchsreihe mit Muskelgewebe vom Menschen (8 Gele einer Auftrennung ein und derselben Gewebeprobe)

wandert ist. Es war festzustellen, $\mathrm{da} ß$ sich die Intensität der Bande je nach dem Grad der Blutbeimengung des excidierten Muskelstückes änderte, was ein Hinweiș auf Hämoglobin gibt. Diese bräunlich-rote Eiweißbande stellt nach der Anfärbung mit Amidoschwarz die stärkste Fraktion des Proteinmusters dar und zeigt eine positive Peroxydasereaktion. Bei fast allen diesen


Abb. 3

Elektropherogramm von Muskelgewebe des Menschen. Schematische Darstellung der Fraktionen mit Angabe der mittleren Wanderungsgeschwindigkeiten ( $m_{r}$-Werte) und densitometrische Kurve

(Vergrößerung: Originalgel: Original-Densitometerkurve $=1: 2$; verwendet wurde der Graukeil der Fa. Joyce, Loebl \& Co. Ltd.)
ing 
Nachweisreaktionen zeigte sich eindeutig eine Zweiteilung dieser Bande (Hâmoglobin neben Myoglobin?), die allerdings innerhalb kurzer Zeit zu einer breiten Fraktion verschmolz. Am kathodischen Ende des Trenngels befinden sich konstant 9-10 feine Banden, die sich gegen eine immer vorhandene Hintergrundfärbung jedoch scharf abgrenzen lassen.

Es folgen regelmäßig 2 starke, sich intensir anfärbende Proteinfraktionen und 3-4 schwāchere Banden. Unterhalb der oben bereits erwähnten stārksten Fraktion, cie sich etwa in der Nitte des Trenngels befindet, folgen 2-3 schwache Fraktionen, denen sich im anodischen Teil des Trenngels regelmāßig 2 breite, intensiv gefärbte Banden anschließen. Dieses Proteinmuster war in erstaunlich hohem $\mathrm{MaBe}$ immer reproduzierbar (s. Abb. 2).

Um dieses Proteinmuster zahlenmäßig zu erfassen, bedienten wir uns, wie riele andere Autoren, mangels anderer Differenzierungsmöglichkeiten, der relativen Beweglichkeit ( $m_{r}$-Werte) der einzelnen Eiweißfraktionen (Abb. 3).

In dieses Schema sind alle Fraktionen eingezeichnet, die bei optimaler Elektrophorese in allen vier Fällen gefunden worden sind. Die eingeklammerten $\mathrm{m}_{\mathrm{r}}$-Werte kennzeichnen Fraktionen, die sich nur vereinzelt darstellien.

Für die densitometrische Auswertung der Gelsäulen wurde das „Double Beam Recording Mikrodensitometer AK IIIC" der Firma Joyce, Loebl \& Co., Ltd.-England, benützt.

Eine quantitative Auswertung der erhaltenen Kurven und somit eine Aufteilung der Proteinfraktionen in relatiren Prozentangaben ist mit einem so großen Fehler $(10-20 \%)$ behaftet, daß man daraus keine objektiv rerwertbaren Aussagen machen kann.

\section{Diskussion}

Der elektrophoretischen Analysse von Körperflüssigkeiten, speziell des Serums, zur Aufklärung pathologischer Verhältnisse steht eine relativ große Mlenge an Untersuchungsmaterial zur Verfügung. Andere Voraussetzungen treffen wir dagegen bei der elektrophoretischen Untersuchung von bioptisch gewonnenen Gewebeproben, z. B. des Muskels an. Die Erfassung von Proteinrerānderungen bei pathologischen Prozessen im Bereich des Muskelgewebes im Rahmen der klinischen Diagnostik ist von größtem Interesse. Doch ist es nicht immer leicht, genügend große Mengen an Muskel zur Herstellung von Extrakten zu erhalten, wenn es sich um bioptisches Material handelt, das auch für histologische und histochemische Untersuchungen dienen soll.

Mikroelektrophoresen, wie sie von Felgenhauer (8) oder PUN und Lombrozo (18) angegeben wurden, lösen dieses Problem auch nicht, da sie in der Methodik kompliziert sind und nur ganz speziellen Untersuchungen rorbehalten bleiben.

Die Herstellung von Gewebsextrakten bringt neben dem zusätzlichen Zeitaufwand noch weitẹre Unsicherheits- faktoren mit sich, die von der Exaktheit der Zusammensetzung und der Ionenstärke des zur Extraktion rerwendeten Puffers, sowie der Homogenisierung und Zentrifugation des Gewebes abhāngen. Aus diesen Gründen bietet die unmittelbare Einbringung von Gewebsschnitten in ein geeignetes Elektrophoresesystem die Möglichkeit, eine Eiweißtrennung mit der geringen rorhandenen Substanizmenge durchzuführen und den Arbeitsaufwand auf ein Minumum zu beschrānken.

Die direkte Gewebslelektrophorese ist bis jetzt lediglich in einigen wenigen Fällen in der Lireratur beschrieben worden. So erwāhnten Wresie (19) und Dents (20) die Möglichkeit der unmittelbaren Einbringung von bioptischem Gewebsmaterial in Agargel, während diesbezügliche Hinweise für die Stärke- und Polyacrylamidgel-Elektrophorese bis jetzt fehlen. Am nächsten kommt ihr die Methode von Matorsty (21), der für seine Untersuchungen lyophilisiertes Gewebe als Pulver zwischen zwei Schichten von feinen Glasperlen mit in das großporige Gel einpolymerisierte.

Der Ausgangspunkt unserer Überlegungen war, mit den gegebenen Möglichkeiten eine schnelle und einfache elektrophoretische Trennung zu erreichen, die dennoch exakte und reproduzierbare Ergebnisse liefern sollte. Eine wesentliche Rolle spielte dabei die Forderung, da $B$ das bioptische Material, das zur histologischen und histochemischen Untersuchung entnommen wurde, auch noch für die Elektrophorese ausreichen mußte. Die Vorteile einer kombinierten histologischen, histochemischen und elektrophoretischen Auswertung einer extrem dünnen Muskelschicht liegen klar auf der Hand. Die zweite Bedingung, die erfüllt sein sollte, war die Anwrendbarkeit der Elektrophoresetechnik auch für die blinische Auswertung. Mit der Disc-Elektrophorese gelang es uns, diesen Forderungen zu genügen und mit der bei einer einmaligen Muskelbiopsie gewonnenen Substanzmenge eine brauchbare und gut reproduzierbare direkte Getvebselektrophorese durchzuführen.

Bei den ersten Versuchen konnte gegen Ende der Elektrophorese eine teilweise Verflüssigung des Sammelgels beobachtet werden, die auf der relativ starken Erwärmung dieses Gelbereiches beruhte. Darauf lāßt sich ein mehr oder weniger starkes Absinken der Schnitte und eine unscharfe Trennung zurückführen. Durch Benutzung unseres Disc-Elektrophorese-Gerätes wurde eine gute Kühlung aller Gelabschnitte, ausgenommen den Bereich der Schicht kathodenwärts der Probe, erreicht. Die oben genannten Erscheinungen ließ̉en sich so vermeiden. Die Auftrennung war vollstāndiger und gleichmäßiger. Eine Standardisierung in bezug auf die genaue Bestimmung der zur Auftragung gekommenen Proteinmenge ließe sich relativ leicht erreichen, indem man mit einem dünnen Korkbohrer aus dem gefrorenen Gewrebestück einen Zylinder ausstanzt und zum Schneiden im Kryostaten verwendet. Somit hat man bei Verwendung einer gleichen Anzahl von Gewebsschnitten eine bestimmbare und immer gleichbleibende Proteinmenge. 


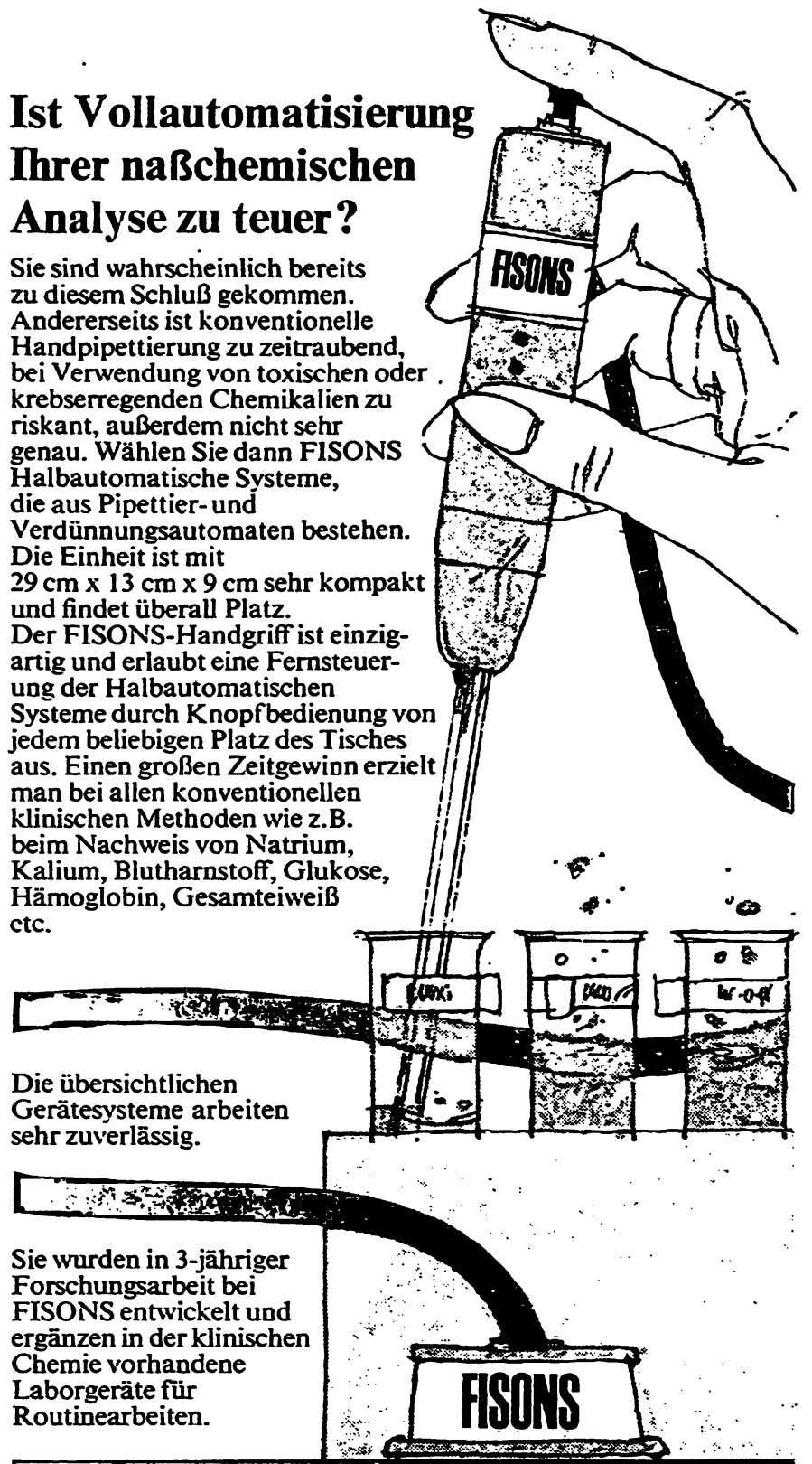

and Lisons Limited International Divion.



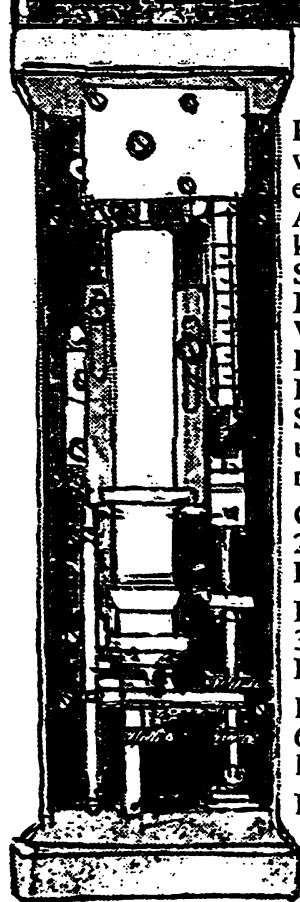

Prüfen Sie, vie dieses einfache System Ihre Arbeit erleichtern kann. Fordern Sie technische Beschreibungen an. Wegen weiterer Einzelheiten und einer Demonstration wenden Sie sich bitte an eines unserer Verkaufsbüros mit Kundendienst: Günther Schmidt, 2Hamburg 68, Postfach 680104

Hans JoachimKleinfeld, 3 Hannover 1 , Postfach 3303

IMA GmbH \& Co. 63 Giessen, Marburgerstraße 81. Bender \& Hobein $\mathrm{GmbH}$, Karlsruhe, München, Freiburg.

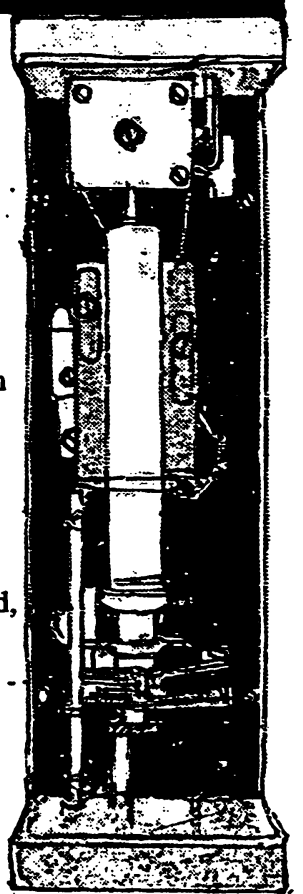

(2.) FSA 17



10000 Mettler-Analysenund Präzisionswaagen

hat unsere Gesellschaft von April 1950 bis Mai 1968 in Deutschland verkauft. Ein beispielloser Erfolg! Wir haben in den Jahren 1950 bis 1957 die Marke Mettler auf dem deutschen Markt eingeführt und fast zwei Jahrzehnte lang an der starken Verbreitung moderner Feinwaagen maßgebend mitgewirkt. Wir beraten und beliefern Sie gern.

\section{Autorisierte Mettler-Vertretung:}

\section{Colora Messtechnik GmbH} 7073 Lorch/Württ., Postfach 5 T (07172) 6041, FS 07-248886

Technische Büros (Verkauf und Kundendienst): 1000 Berlin 30, KurfūrstenstraBe 84, T 135200 2000 Hamburg 19, OsterstraBe 63, T 4006 06, FS 02-12 947 3000 Hannover, An der Tiefenriede 45, T 884500 4000 Düsseldorf, Kronprinzenstr. 62, T 17860 , FS 08-587 253 6000 Frankfurt/M., Rōderbergweg 4-6, T 446031, FS 04-11 216 7073 Lorch/Württ., Barbarossastr. 3, T 60 41, FS 07-248 886 8000 Mūnchen 2, Dachauer StraBe 175, T 5169858

\section{BOIOR}




\section{Die Injektion}

Technik - Praxis - Komplikationen. Von Dr. med. Dr. med. dent. Joachim Gabka. Groß-Oktav. XII, 205 Seiten. Mit 73, z. TI. mehrfarbigen Abbildungen. 1968. Plastik flexibel DM 20,-

\section{Allgemeine Mikrobiologie}

Leitsätze für Studierende und Ärzte. Von Prof. Dr. med. UIrich Schneeweiß. Unter Mitarbeit vonEva-Maria Fabricius. Mit 111 Abbilbungen und 47 Tabellen. XII, 343 Seiten. 1968. Plastik flexibel DM 20,-

\section{Spezielle Mikrobiologie}

Leitsätze für Studierende und Ärzte. Von Prof. Dr. med. Ulrich Schneeweiß. Unter Mitarbeit von Eva-Maria Fabricius. Groß-Oktav. Mit 158 Abbildungen und 39 Tabellen. XII, 477 Seiten. 1968. Plastik flexibel DM 30,-

\section{Praktikum der Physiologischen Chemie}

von Dr. Peter Siegmund, Dr. Dr. Ernst Schütte und Dr. Friedrich Körber. Mit 49 Abbildungen und vielen Formeln. Oktav. XVI, 287 Seiten. 1968. Plastik flexibel DM 19,80

\section{Lehrbuch der Physiologischen Chemie}

von Prof. Franz Leuthardt. Begr. von S. Edlbacher. 15., neubearbeitete Auflage. Groß-Oktav. Mit 76 Abbildungen, 1 Bildtafel. XVI, 912 Seiten. 1963. Plastikeinband DM 42, -

\section{Praktische Gynäkologie}

Für Studierende und Ärzte von Prof. Dr. med. Dr. phil. W. Pschyrembel. 4., überarbeitete und erweiterte Auflage. Oktav. Mit 503, teils mehrfarbigen Abbildungen. XXIV, 642 Seiten. 1968. Plastikeinband DM 54,-

\section{Praktische Geburtshilfe}

Für Studierende und Ärzte von Prof. Dr. med. Dr. phil. W. Pschyrembel. 12./13. Auflage. Oktav. Mit 502, davon 3 mehrfarbigen Abbildungen. XXIV, 807 Seiten. 1967. Plastikeinband DM 65, -

\section{Anatomie des Menschen}

Für Studierende und Ärzte, dargestellt nach systematischen, topographischen und praktischen Gesichtspunkten von Prof. Dr. med. et phil. A. Waldeyer, unter Mitarbeit von Ursula Waldeyer. 2 Teile. Groß-Oktav. Ganzleinen.

1. Teil: Allgemeine Anatomie, Rücken, Bauch, Becken, Bein. 6., überarbeitete Auflage. Mit 335, meist farbigen Abbildungen. XVI, 447 Seiten. 1969. DM 48, -

2. Teil: Kopf und Hals, Auge, Ohr, Gehirn, Arm, Brust. 4. und 5., überarbeitete Auflage. Mit 447, zum großen Teil farbigen Abbildungen. XVI, 602 Seiten. 1967. DM 62, -

\section{Augenheilkunde}

Leiffaden für Studium und Praxis von Prof. Dr. med. Hugo Gasteiger. 2., neubearbeitete und erweiterte Auflage. Groß-Oktav. Mit 277, zum großen Teil mehrfarbigen Abbildungen. XVI, 303 Seiten. 1963. Ganzleinen DM 45, -

\section{Ohren-, Nasen-, Rachen- und Kehlkopfkrankheiten}

Begr. von A. Knick. 35./36., neu bearbeitete und ergänzte Auflage von o. Prof. Dr. Gerhard Eigler. Groß-Oktav. Mit 164, teilweise mehrfarbigen Abbildungen. XVI, 261 Seiten. 1966. Plastikeinband DM 28, - 
Uber die Identifikation der getrennten Eiweißfraktionen sind Untersuchungen im Gange, sie werden an anderer Stelle mitgeteilt. Vergleiche mit Disc-Elektrophoresen von Muskelgewebc des Menschen in Polyacrylamidgel konnten nicht angestellt werden, da entsprechende Versuche bis jetzt fehlen. Aus Untersuchungen mit Muskclextrakten in Agargel von BAUER (22), sowie in
Stärkegel von HugHes $(1,23)$ können infolge der zu unterschiedlichen Methoden keine Rückschlüsse für cine zusätzliche Identifizicrung der Proteinfraktionen gezogen werden, doch sind durch die Anwendung der direkten Gewebselektrophorese in Polyacrylamidgel die frühcr beobachteten Schwierigkeiten bcseitigt.

\section{Literatur}

1. Hugres, B. P., Clin. Chim. Acta Amstcrdam 6, 794 (1961). 2. Raymond, S. und I. Weintraub, Science Washington 130 , 711 (1959). - 3. Davis, B. J., Ann. N. Y. Acad. Sei. 121, 404 (1964). - 4. Ornstern, L., Ann. N. Y. Acad. Sci. 121, 321 (1964). 5. Poulik, M. D., Naturc London, 180, 1477 (1957). - 6. ANrz, W. K., Inaugural-Disscrtation, München (1968). - 7. MAURer, H. R., Disk-Elcktrophorcsc, Waltcr de Gruyter \& Co., Berlin (1968). - 8. Felceninuer, K., Biochim. biophysica Acta Amsterdam, 133, 165 (1967). - 9. MaJzer, J. V., Science Washington, 151, 988 (1966). - 10. Chrambach, A., R. A. Reisfeld, M. Wyckofr und J. ZacCari, Analytic. Biochem. 20, 150 (1967). 11. MeYer, T. S. und B. L. Lamberts, Biochim. biophysica Acta Amsterdam 107, 144 (1965). - 12. ST. GROTH, P. S. De., R. G. Webstrer und A. DATnNer, Biochim. biophysica Acta Amsterdam, 71, 377 (1963). - 13. Scrware, C., Analytic. Biochem. 17,
201 (1966). - 14. Schrauwein, J. A. M., J. Chromatogr. 15 256 (1964). - 15. MAUReR, H. R., dicsc Z. 4, 85 (1966). - 16. NAGY, L. K., B. Rocerson und N. Tomkuss, Nature London 212, 923 (1966). - 17. Dietz, A. A. und T. Lubrano, Analytic. Biochem. 20, 246 (1967). - 18. PUN, J. Y. und L. LOMвro\%O, Analytic. Biochem. 9, 9 (1964). - 19. WIEME, R. J., Agar Gel Electrophoresis, Elsevier Publishing Company; Amstcrdam, London, New York (1965). - 20. Denis, L. J., G. R. Prourr, Va. Richmond, M. van Sande und K. van Camp, J. Amct. Med. Ass. 178, 1093 (1961). - 21. Matolsty, A. G. und M. N. MArolsty, J. Investigat. Dcrm. Baltimorc 41, 255 (1963). - 22. Bauer, H., F. Glötzaner, I. Schiwarze und K. Schmalaach, Klin. Wschr. 42, 1198 (1964). - 23. Hugries, B. P., Biochcm. Biophys. Res. Commun. 1, 194 (1959).

Priv.-Do\%. Dr. F. Mittclbach 800 München 15 Ziemssenstr. 1 